\title{
Prevalence of depression among HIV-positive patients treated with antiretrovirals at different stage of infection
}

\author{
Yasir Alvi, Najam Khalique, Anees Ahmad, Haroon S. Khan, Nafis Faizi
}

Department of Community Medicine, Jawaharlal Nehru Medical College \& Hospital, Aligarh Muslim University (AMU), Aligarh, India

\begin{abstract}
Introduction: Depression in human immunodeficiency virus (HIV) and acquired immune deficiency syndrome (AIDS) is common and can have far-reaching implications in patients receiving antiretroviral therapy (ART). A high prevalence of depression has been reported in people living with HIV/AIDS (PLHA). With the improvement in the management of HIV/AIDS patients, depression is becoming a common medical problem and interferes with ART during HIV infection. This study was conducted with the objectives to determine the prevalence of depression and to study its relationship at different stages of infection (WHO staging) and disease progression.

Material and methods: About 434 HIV-positive patients on ART from Anti-Retroviral Treatment Center in Aligarh, India were interviewed using pre-tested predefined questionnaire PHQ-9. The data was analyzed using SPSS20 and a $p$-value of less than 0.05 was considered statistically significant.

Results: We found that 70/434 (16.1\%) ART patients suffered from depression, with 13/434 (3\%) suffering from moderate and moderately severe depression. In addition, depression was found to be greater among higher stage of the infection. About $9 / 70$ (12.9\%) patients with depression were found to be in WHO stage III or IV as compared to $23 / 364$ (6.3\%) of non-depressive patients in same WHO stage. Depression was also significantly related with worsening of disease progression, with the mean improvement in CD4 levels with treatment being only $88.54 \pm 94.91$ in patients suffering from moderate and moderately severe depression, as compared to $197.31 \pm 169.84$ in non-depressive patients.

Conclusions: Depression has a significant relation with a higher stage of infection and disease progression of the HIV-infected respondents. Therefore, screening for depression is essential and should be incorporated in primary patient care.
\end{abstract}

HIV AIDS Rev 2018; 17, 4: 243-248

DOI: https://doi.org/10.5114/hivar.2018.80255

Key words: depression, CD4, HIV, antiretroviral therapy, PHQ.

\section{Introduction}

Depression is one of the leading causes of disease burden and may lead to loss of quality of life with social and economic dysfunction, causing misery to both the sufferers and their families. Major depression is the second most com-

mon reason of disability worldwide [1]. Despite the paucity of studies, as per the reports available in India, no less than one in every twenty Indians suffer from depression [2]. In the presence of comorbidities like human immunodeficiency virus (HIV) and acquired immune deficiency syndrome (AIDS), the risk of acquiring depression further increases [3].

Article history:

Received: 28.10.2017

Received in revised form: 09.04.2018

Accepted: 11.04.2018

Available online: 20.11.2018
International Journal of HIV-Related Problems

HIV \& AIDS

R e v i e w 
Previous studies have documented a high prevalence of depression among people living with HIV/AIDS (PLHA), varying from $8.1 \%$ to $67.3 \%$, quite higher than overall prevalence of depression [2-11]. Among many comorbidities associated with HIV/AIDS patients, depression ranks higher than most of the others. Depression and HIV/AIDS are also projected to be two leading causes of illness worldwide, and their combined presence could have detrimental effects on the disease management [12]. With the advent of highly active antiretroviral therapy (HAART), the lifespan of people living with HIV/ AIDS (PLHA) has increased. The threat of depression in this prolonged lifespan is real and can have far reaching implications on the prognosis and treatment. In some settings, as high as one in three persons with HIV could have major depression [13]. The cause of depression in PLHA could be due to immune mediated injury on the nervous system or premature cortical atrophy [14]. It could also be due to the stress of illness, maladjustment to difficult life events like unemployment and marriage or stigma, and discrimination related to AIDS [15, 16]. Regardless of the cause, depression has a direct and indirect influence on the treatment outcomes of HIV/AIDS. A higher mortality is associated with depression and advanced HIV disease [5, 17]. Comorbid depression has shown to influence the decline in CD4 cell counts as well as increase in the plasma viral load $[18,19]$. Depression could be life threatening and is associated with high-risk behavior and quicker progression to immunodeficiency in PLHA [20]. Considering the high burden of HIV/AIDS patients in India and the paucity of studies in this regard, this study was conducted with two objectives: 1 . To determine the prevalence of depression among PLHA being treated with anti-retroviral therapy (ART) in Center in Aligarh; 2. To find out the relationship between depression and different stage of infection as per WHO staging and disease progression in terms of $\mathrm{CD} 4$ cell count changing.

\section{Material and methods}

\section{Study design and setting}

This was a cross-sectional study, conducted from July 2015 to June 2016. All patients who were on antiretroviral treatment at Anti-Retroviral Treatment (ART) Centre of Jawaharlal Nehru Medical College, Aligarh, Uttar Pradesh were selected for the study. Incidentally, Aligarh is one of the populated districts of the state of Uttar Pradesh, which itself is the most populated state of the country, the population being $5^{\text {th }}$ highest in the world (when treated as a country) [21]. The ART Centre of Aligarh is one of the oldest ART Centers in India, which now has a total of 528 ART Centers [22]. The ART Centre of Aligarh also caters to the patients from neighboring districts with two link ART Centre attached to it.

\section{Study population}

The sample size of the study was based on the objective of determining the prevalence of depression. Based on the estimation of prevalence, the formula, $n=z^{2} p(1-p) / d^{2}$ was used, where $z$ was taken as 1.96 , based on the level of significance $(\alpha=0.05)$. The anticipated prevalence $p$ was taken as $49 \%$, based on a previous study conducted in 2012 in India [11], whereas the absolute precision was taken as $10 \%(d)$. Thus, the sample size came out to be 400 , which was adjusted for presumed non-response rate of $10 \%$, making it 440 . All the adult patients ( $>18$ years) on ART were eligible for the study on selection, based on the sampling frame. All the included patients were selected for the study after their written consent. The exclusion criteria were: 1 . Patients with duration of ART less than six months; 2. Patients with the acute medical condition during the study; 3 . Any past or family history of the psychiatric condition; 4 . Any bereavement or significant loss of property or sickness/death of any close relatives within the past 3 months.

\section{Sampling design}

The sample population was selected using the systematic random sampling. The sampling frame of the study was made from the list of the patients reporting to the ART Centre on the day of data collection. The interview was done thrice a week on alternate days, which were altered in consecutive weeks in order to counter the bias. With the average daily attendance of the ART Centre being 60, and with an aim to interview six patients in a day, the sampling interval was calculated to be 10. The first number was chosen randomly, after which every tenth patient in the sampling frame was selected for the data collection. If the selected patient was not eligible for this study or was already interviewed earlier then the next patient from the sampling frame was selected for the interview.

\section{Study instruments}

The study tool was a pre-tested proforma, using the local (Hindi) language version of Physical Health Questionnaire (PHQ)-9 to measure depression. The PHQ-9 consists of nine measures, on which the DSM-IV depressive disorders diagnosis is based [23]. In addition, it also measures the severity of depression, and was specially developed to screen and diagnose depression at primary care settings [23]. Each of the nine items of this questionnaire is scored from 0 ('not at all') to 3 ('nearly every day'). Total score ranges from 0 to 27 and severity of depression is classified as: none or minimal (0-4), mild (5-9), moderate (10-14), moderately severe (15-19), and severe (20-27). Sensitivity and specificity, for the local (Hindi) version of PHQ-9, which was used in the study in diagnosing depression was reported to be $99 \%$ and $90 \%$, respectively [24]. For the different stage of infection, WHO staging and current CD4 levels were determined. WHO staging is a clinical staging of HIV/AIDS patients based on HIV-associated symptoms. It has four stages: asymptomatic, mild symptoms, advance symptoms, and severe symptoms represented by roman numbers I, II, III, and IV, 
respectively [25]. CD4 cells count was assessed by measuring the absolute number (per $\mathrm{mm}^{3}$ ) according to the WHO norms [25]. The CD4 levels were measured twice - one for current values and the other at the start of treatment. The progression of the disease was measured by calculating the difference between current CD4 values with the CD4 levels that were present at the start of the treatment.

\section{Data management and statistics}

The data was entered and analyzed in Statistical Package for Social Sciences (SPSS) version 20 [26]. The categorical variables and proportions were expressed as the percentage (\%) along with $95 \%$ confidence interval. The $95 \%$ confidence intervals for proportions were calculated using the AgrestiCoull or modified Wald's method. Statistical significance was tested by the $\chi^{2}$ test or Fisher's exact test, as applicable [27]. Continuous variables were expressed in means and standard deviations (SD), and Student's $t$-test was used for statistical significance of independent samples. Analysis of variance (ANOVA) was applied, wherever applicable with Fisher's least significant difference (LSD) test for post-hoc assessments. Probability $(p)$ was calculated to test for statistical significance at the $5 \%$ level of significance $(p<0.05)$.

\section{Ethics}

The study was first approved by an academic board of studies, followed by Institutional Ethics and Research Advisory Committee, JN Medical College, AMU, Aligarh. A written consent was taken from each selected participant to confirm willingness, after prior approvals from the ART Centre. The actual study was preceded by an individual rapport building session, where the purpose of the research and its implications were discussed. The participants were ensured about the privacy and confidentiality of the research and this was maintained during the entire duration of the study. The interviewer of the study for all the participants was the same investigator. Appropriate health education and adequate counselling were provided to all the patients after the interview. Those found to have depression were encouraged to visit the psychiatry clinic of the Medical College and were assisted if agreed.

\section{Results}

Out of 440 patients selected, only 434 completed the interview. About 16.1\% (95\% CI: 13.0-19.9; 70/434) of the patients were found to be suffering from depression. Figure 1 and Table 1 depict the prevalence of current depression among the patients treated with ART. Further, it was found that among the patients suffering from depression, most of them had mild depression (81.4\%) followed by moderate and moderately severe depression $(14.3 \%$ and $4.3 \%$, respectively) (Table 1).

Additionally, the depression was compared with clinical severity. We began this analysis by testing the presence or absence of depression with WHO staging. About nine (12.9\%) patients suffering from depression were found to be present in stage III and IV, 31 (44.3\%) in stage II, and $30(42.9 \%)$ in stage I. Among the patients without depression, $23(6.3 \%)$ were in stage III and IV, $122(33.5 \%)$ in stage II, and $219(60.2 \%)$ in stage I. Thus, the severity of the infection in terms of WHO stages was found to be significantly higher in patients suffering from depression $\left(\chi^{2}(2,434)=8.401\right.$; $p<0.05)$. The prevalence of depression was also significant when grades of depression from absence to severity

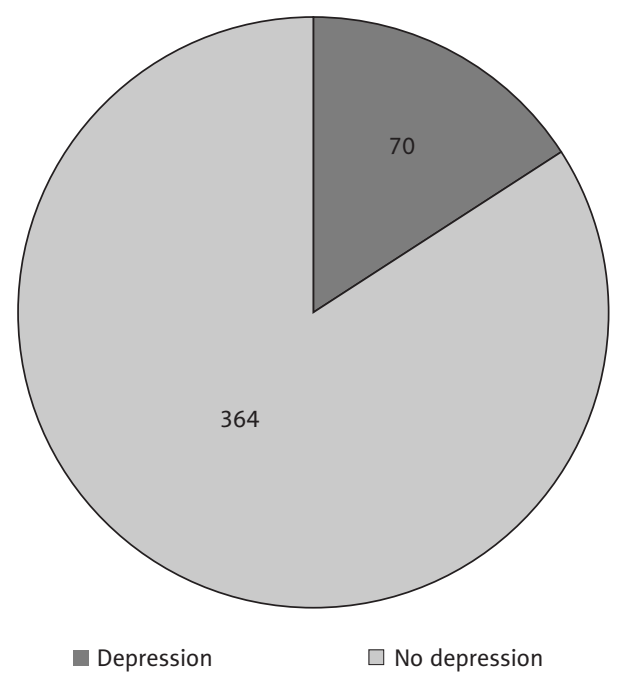

Figure 1. Distribution of patients with depression attending antiretroviral therapy center

Table 1. Frequency distribution of patients attending antiretroviral therapy center on basis of grades of depression

\begin{tabular}{l|c|c|c}
\hline Pattern of depression & Frequency & Percentage & $95 \% \mathrm{Cl}$ \\
\hline No depression & 364 & $83.9 \%$ & $80.1-87.0$ \\
\hline Depression & 70 & $16.1 \%$ & $13.0-19.9$ \\
\hline Mild & $57(81.4 \%)$ & $13.1 \%$ & $10.3-16.7$ \\
\hline Moderate & $10(14.3 \%)$ & $2.3 \%$ & $2.3-12.0$ \\
\hline Moderately Severe & $3(4.3 \%)$ & $0.7 \%$ & $0.06-1.36$ \\
\hline Total & 434 & $100 \%$ & \\
\hline
\end{tabular}


Table 2. Frequency distribution of grades of depression according WHO staging among patients being treated with antiretroviral drugs

\begin{tabular}{l|c|c|c|c|c|c}
\hline \multirow{2}{*}{ WHO stage } & \multicolumn{5}{|c}{ Depression } \\
\cline { 2 - 7 } & \multicolumn{2}{|c|}{ No depression } & \multicolumn{2}{c}{ Mild depression } & \multicolumn{2}{c}{ Moderate and moderately severe } \\
\cline { 2 - 7 } & Freq. (\%) & $95 \% \mathrm{Cl}$ & Freq. (\%) & $95 \% \mathrm{Cl}$ & Freq. (\%) & $95 \% \mathrm{Cl}$ \\
\hline $\mathrm{I}$ & $219(60.2)$ & $55.1-65.1$ & $26(45.6)$ & $33.4-58.4$ & $4(30.8)$ & $12.4-58.0$ \\
\hline $\mathrm{II}$ & $122(33.5)$ & $28.9-38.5$ & $24(42.1)$ & $30.2-55.0$ & $7(53.8)$ & $29.1-76.8$ \\
\hline III and IV & $23(6.3)$ & $4.2-9.3$ & $7(12.3)$ & $5.8-23.6$ & $2(15.4)$ & $3.1-43.5$ \\
\hline Total & $364(83.9)$ & $80.1-87.0$ & $57(13.1)$ & $10.3-16.7$ & $13(3.0)$ & $1.7-5.1$ \\
\hline F=9.360. & & & & &
\end{tabular}

$F=9.360 ; p$ value $<0.05$

Table 3. Comparison of the grades of depression with CD4 count among patients being treated with antiretroviral drugs

\begin{tabular}{|c|c|c|c|c|c|c|c|c|}
\hline & \multirow{2}{*}{ Freq. } & \multirow{2}{*}{ Mean } & \multirow{2}{*}{ SD } & \multicolumn{2}{|c|}{$95 \% \mathrm{Cl}$} & \multirow{2}{*}{ df } & \multirow{2}{*}{$\mathrm{F}$} & \multirow{2}{*}{$p$ value } \\
\hline & & & & Lower & Upper & & & \\
\hline \multicolumn{9}{|c|}{ CD4 count at start of antiretroviral therapy } \\
\hline Moderate and Moderately severe & 13 & 189.69 & 61.65 & 152.44 & 226.94 & \multirow{3}{*}{2} & \multirow{3}{*}{2.061} & \multirow{3}{*}{0.129} \\
\hline Mild & 57 & 228.60 & 99.81 & 202.11 & 255.08 & & & \\
\hline No & 364 & 198.98 & 106.90 & 187.96 & 210.00 & & & \\
\hline \multicolumn{9}{|l|}{ Current CD4 count } \\
\hline Moderate and Moderately severe & 13 & 278.23 & 110.89 & 211.22 & 345.25 & \multirow{3}{*}{2} & \multirow{3}{*}{3.340} & \multirow{3}{*}{0.036} \\
\hline Mild & 57 & 357.70 & 172.87 & 311.83 & 403.57 & & & \\
\hline No & 343 & 393.07 & 181.34 & 373.81 & 412.33 & & & \\
\hline \multicolumn{9}{|l|}{ Progression of CD4 count* } \\
\hline Moderate and Moderately severe & 13 & 88.54 & 94.91 & 31.18 & 145.89 & \multirow{3}{*}{2} & \multirow{3}{*}{6.390} & \multirow{3}{*}{0.002} \\
\hline Mild & 57 & 129.10 & 151.43 & 88.92 & 169.28 & & & \\
\hline No & 343 & 197.31 & 169.84 & 179.27 & 215.35 & & & \\
\hline
\end{tabular}

*Progression of CD4 count was measured by calculation the difference between current $C D 4$ values and the CD4 value at start of antiretroviral therapy

were compared with WHO staging (Table 2). Consequently, we compared the current CD4 levels of the patients with the presence of depression. We analyzed the differences between the presence of depression and CD4 cells count. On applying independent $t$-test, a significant difference was found between the presence of depression and current CD4 cells count $(t(411)=-2.138 ; p<0.05)$. However, this was not found with CD4 cells count at the start of treatment $(t(432)=1.633 ; p>0.05)$. Further, we compared the grades of depression and current CD4 cells count using ANOVA. With increasing severity of depression, the severity of the infection, in terms of lower CD4 cell count, was found to be increasing (Table 3 ).

On applying post hoc LSD test, a significant difference was found between current CD4 cells count of patients with no depression and patients suffering from moderate and moderately severe depression $(p<0.05)$. As far as depression and progression of the disease are concerned, the progression of CD4 levels from the start of the treatment to current CD4 levels was used. It was found that the mean of CD 4 cell count progression among those suffering from moderate and moderately severe depression $(\mathrm{M}=88.54$; $\mathrm{SD}=94.91)$ was lower than those with mild depression $(\mathrm{M}=129.10 ; \mathrm{SD}=151.40)$ and no depression $(\mathrm{M}=197.31$; $\mathrm{SD}=169.84)$, and this was statistically significant on ANOVA $(\mathrm{F}(2,413)=6.390$; $p<0.05)$. On post hoc LSD test, a significant difference was found between mean progression of CD4 cells count of no depression with mild depression $(p<0.05)$ as well as moderate and moderately severe depression $(p<0.05)$ (Table 3). However, no significant difference existed between mean progression of CD4 cells count of mild depression with moderate and moderately severe depression $(p>0.05)$.

\section{Discussion}

We conducted this study with the objective to determine the prevalence of depression and its relationship with different stage of infection and disease progression. The prevalence of depression was $16.1 \%$ in this study, similar to $14.0 \%$ in Uganda and $15 \%$ in the United States $[4,5]$. 
Another systemic review conducted in 2014 in sub-Saharan African countries found the pooled prevalence of major depressive disorder to be $14.5 \%$ (95\% CI: 8.9 21.2) [6]. Among studies conducted in India, the prevalence was found to be $16 \%$ in Maharashtra, South-West Indian state [7]. Some Indian studies also report higher rates of depression, $49 \%$ to $67 \%$ [8-11]. Further comparison of the prevalence of depression between our and these studies is difficult due to different study instruments diagnosing depression, methodologies, study population, and clinical settings. This prevalence of depression in patients treated with ART is higher than the prevalence in the general population [2]. This high prevalence is supported by another study conducted in the Northern state of India, emphasizing the need for early diagnosis and prompt treatment of depression in HIV patients [10]. Encouragingly, the proportion of mildly depressed patients was higher in our study: 57/70 (81.9\%), predicting a good value for money and efforts in this direction, as a periodic screening of HIV patients for depression can easily detect and manage mild depression cases. In our study, the prevalence of depression increased with higher stage of infection in terms of higher WHO stage of the infection as well as with lower current CD4 levels. The association between depression and higher WHO stage has been documented previously by studies in India and Tanzania; however, no such association was reported by the meta-analysis conducted by Ciesla et al. $[3,8,28]$. Nevertheless, unlike our study, the study by Ciesla et al. classified the patients in only two HIV stages such as asymptomatic and symptomatic [3]. The relationship of lower CD4 levels and depression has been documented from different parts of the World [5, 2931]. Although, some studies failed to find a significant association between depression and CD4 cells count $[9,10]$. The progression of HIV infection was poorer in patients suffering from depression in our study. Though, the relationship between depression and the severity of the infection have been widely studied, scientific researches are scarce from this particular geographical area. In fact, to the best of our knowledge, this study is the first study that compares these two important variables from Southeast Asia. However, depression leading to a poor progression of the HIV infection has been documented elsewhere in the World [5, 17-19]. Therefore, the poor progression of the disease due to depression, which is often unknown and undetected, is a cause of concern for public health management of HIV/AIDS. Our study also had a few limitations. Firstly, it was a cross-sectional study completed in hospital settings, therefore having a restricted generalizability. Though most of the patients of HIV/AIDS in India $(997,000$ as on September 2016) avail treatment from the 528 ART Centres only [22]. Secondly, we refrain from any causal association because of the study design, as the temporality cannot be ascertained. Nonetheless, we have tried our best to measure the progression of the disease through the current CD4 levels and the levels at the start of treatment.

\section{Conclusions}

The prevalence of depression in HIV/AIDS patients on antiretroviral therapy in Aligarh was $16.1 \%$, with $18.6 \%$ suffering from moderate to severe depression. A higher prevalence of depression was seen with worsening of the HIV infection in terms of current WHO staging and CD4 count. We believe that our results reflect three vital concerns: 1. The prevalence of depression in HIV/AIDS patients is higher than the uninfected general population, and is mostly unknown and undetected; 2. Prevalence of depression is higher among higher stage of the infection; 3. Depression is vital to detect as early as possible as it can worsen the prognosis of the disease. Therefore, a regular screening and follow-up of patients on ART, as others have recommended, should be considered by the National AIDS Control Program [6, 20, 31].

\section{Conflict of interest}

The authors declare no potential conflicts of interest with respect to the research, authorship, and/or publication of this article.

\section{References}

1. Ferrari AJ, Charlson FJ, Norman RE, et al. Burden of Depressive Disorders by Country, Sex, Age, and Year: Findings from the Global Burden of Disease Study 2010. PLoS Med 2013; 10: e1001547.

2. National Mental Health Survey of India, 2015-2016. Prevalence, Patterns and Outcomes, Supported by Ministry of Health and Family Welfare, Government of India, and Implemented by National institute of Mental Health and Neurosciences (NIMHANS) Bengaluru: In Collaboration with Partner Institutions; 2015-2016 (cited 2017 May 3). Availabe from: http://indianmhs.nimhans.ac.in/ Documents/Reports/Summary.pdf

3. Ciesla JA, Roberts JE. Meta-Analysis of the Relationship Between HIV Infection and Risk for Depressive Disorders. Am J Psychiatry 2001; 158: 725-730.

4. Kinyanda E, Nakasujja N, Levin J, et al. Major depressive disorder and suicidality in early HIV infection and its association with risk factors and negative outcomes as seen in semi-urban and rural Uganda. J Affect Disord 2017; 212: 117-127.

5. Taniguchi T, Shacham E, Önen NF, et al. Depression severity is associated with increased risk behaviors and decreased CD4 cell counts. AIDS Care 2014; 26: 1004-1012.

6. Tsai AC. Reliability and validity of depression assessment among persons with HIV in sub-Saharan Africa: systematic review and meta-analysis. J Acquir Immune Defic Syndr 2014; 66: 503-511.

7. Das M, Isaakidis P, Van den Bergh R, et al. HIV, multidrug-resistant TB and depressive symptoms: when three conditions collide. Glob Health Action 2014; 7: 24912.

8. Bhatia MS, Munjal S. Prevalence of depression in people living with HIV/AIDS undergoing ART and factors associated with it. J Clin Diagn Res 2014; 8: WC01-4.

9. Unnikrishnan B, Jagannath V, Ramapuram JT, et al. Study of Depression and Its Associated Factors among Women Living with HIV/AIDS in Coastal South India. ISRN AIDS 2012; 2012: 684972.

10. Rai P, Verma BL. A study on depression in people living with HIV/ AIDS in South-West part of Uttar Pradesh, India. South East Asia J Public Health 2015; 5: 12-17. 
11. Selvaraj V, Ross MW, Unnikrishnan B, Hegde S. Association of quality of life with major depressive disorder among people with HIV in South India. AIDS Care 2013; 25: 169-172.

12. Mathers CD, Loncar D. Projections of Global Mortality and Burden of Disease from 2002 to 2030. PLoS Med 2006; 3:e442.

13. Bing EG, Burnam MA, Longshore D, et al. Psychiatric disorders and drug use among human immunodeficiency virus-infected adults in the United States. Arch Gen Psychiatry 2001; 58: 721-728.

14. Bhat AG, Babu R, Abhishekh HA. Prevalence of depression among HIV patients on antiretro viral therapy: A study from India. Asian J Psychiatr 2013; 6: 249-250.

15. Leserman J, Petitto JM, Perkins DO, et al. Severe stress, depressive symptoms, and changes in lymphocyte subsets in human immunodeficiency virus-infected men. A 2-year follow-up study. Arch Gen Psychiatry 1997; 54: 279-285.

16. Page-Shafer K, Delorenze GN, Satariano WA, Winkelstein W. Comorbidity and survival in HIV-infected men in the San Francisco Men's Health Survey. Ann Epidemiol 1996; 6: 420-430.

17. Leserman J. Role of Depression, Stress, and Trauma in HIV Disease Progression. Psychosom Med 2008; 70: 539-545.

18. Ickovics JR, Hamburger ME, Vlahov D, et al. Mortality, CD4 cell count decline, and depressive symptoms among HIV-seropositive women: Longitudinal analysis from the HIV Epidemiology Research Study. JAMA 2001; 285: 1466-1474.

19. Rivera-Rivera Y, Vázquez-Santiago FJ, Albino E, et al. Impact of Depression and Inflammation on the Progression of HIV Disease. J Clin Cell Immunol 2016; 7; doi:10.4172/2155-9899.1000423.

20. US department of Veterans Affairs. Primary care of veterans with HIV: Neurology, psychiatry, and pain; April 2009. Available at: https://www.hiv.va.gov/provider/manual-primary-care/depression.asp (Accessed: 20.06.2017).

21. The Economist. Comparing Indian states and territories with countries: An Indian summary. Available at: http://www.economist. com/content/indian-summary (Accessed: 13.05.2017).

22. National AIDS Control Organization, National Institute of Medical Statistics. Annual report 2016-17; 2017. Available at: http:// naco.gov.in/sites/default/files/NACO\%20ANNUAL\%20REPORT\%202016-17.pdf (Accessed: 4.05.2017).

23. Kroenke K, Spitzer RL, Williams JB. The PHQ-9: validity of a brief depression severity measure. J Gen Intern Med 2001; 16: 606-613.

24. Kochhar PH, Rajadhyaksha SS, Suvarna VR. Translation and validation of brief patient health questionnaire against DSM IV as a tool to diagnose major depressive disorder in Indian patients. J Postgrad Med 2007; 53: 102-107.

25. World Health Organization. WHO Case Definitions of HIV for Surveillance and Revised Clinical Staging and Immunological Classification of HIV-Related Disease in Adults and Children. World Health Organization, Geneva 2007. Available at: http://www. who.int/hiv/pub/vct/hivstaging/en/ (Accessed: 2.06.2015).

26. IBM Corp. Released 2011. IBM SPSS Statistics for Windows, Version 20.0. IBM Corp., Armonk.

27. Epitools Epidemiological Calculators. Available at: http:// epitools.ausvet.com.au/content.php?page $=$ CIProportion (Accessed: 31.06 .2017$)$.

28. Antelman G, Kaaya S, Wei R, et al. Depressive Symptoms Increase Risk of HIV Disease Progression and Mortality Among Women in Tanzania. J Acquir Immune Defic Syndr 2007; 44: 470-477.

29. Kaharuza FM, Bunnell R, Moss S, et al. Depression and CD4 Cell Count Among Persons with HIV Infection in Uganda. AIDS Behav 2006; 10: 105-111.

30. Katz MH, Douglas JM, Bolan GA, et al. Depression and use of mental health services among HIV-infected men. AIDS Care 1996; 8: 433-442.

31. L'akoa RM, Noubiap JJ, Fang Y, et al. Prevalence and correlates of depressive symptoms in HIV-positive patients: a cross-sectional study among newly diagnosed patients in Yaoundé, Cameroon. BMC Psychiatry 2013; 13: 228. 